\title{
Histological evaluation of age-related variations in saphenous vein grafts used for coronary artery bypass grafting
}

\author{
Bartłomiej Perek ${ }^{1}$, Agnieszka Malińska², Michał Nowicki², Marcin Misterski ${ }^{1}$, \\ Danuta Ostalska-Nowicka³, Marek Jemielity ${ }^{1}$
}

\begin{abstract}
1Department of Cardiac Surgery, Poznan University of Medical Sciences, Poland 2Department of Histology and Embryology, Poznan University of Medical Sciences, Poland

${ }^{3}$ Department of Paediatric Cardiology and Embryology, Poznan University of Medical Sciences, Poland
\end{abstract}

Submitted: 18 December 2010

Accepted: 12 February 2011

Arch Med Sci 2012; 8, 6: 1041-1047

DOI: $10.5114 /$ aoms.2012.32412

Copyright (c) 2012 Termedia \& Banach

\begin{abstract}
Introduction: Venous coronary artery bypass grafts (CABG) might undergo a process of arterialization resulting in neointimal formation and medial hypertrophy. It is often followed by critical occlusion of the graft lumen. The aim of the study was to assess histological representative features of saphenous vein reconstruction in aging as well as to establish optimal patients' age limits applicable for optimal selection of grafts.

Material and methods: One hundred and ten patients undergoing venous CABG were divided into 4 age subgroups: (A) 50 years and less, (B) 51-60 years, (C) 61-70 years and (D) > 70 year-old subjects. Distal venous graft segments were saved for an adequate morphometric assay which was followed by suitable statistical analysis.

Results: The entire venous wall thickness as well as its tunica media were found to become significantly thinner between subgroups A and D. The number of smooth muscle cell (SMC) nuclei within the tunica media did not differ between study subgroups. The majority of these nuclei in subgroup $D$ were found, however, to be more elongated than in subgroup A (SMC length/width index in subgroup D was found to be significantly higher than in subgroup A). Conclusions: Progressive, age-related thinning of the venous wall and tunica media as well as SMC nucleus elongation might suggest impairment of SMCs' migration and proliferation rate. Consequently, individuals aged 70 years and over may benefit clinically more from venous CABG than younger patients due to the lower risk of arterialization and occlusion of the graft lumen in the future.
\end{abstract}

Key words: saphenous vein, venous aorto-coronary grafts, smooth muscle cells, nucleus shape, aging.

\section{Introduction}

Coronary artery bypass grafting (CABG) is still a recommended therapeutic procedure in severe multi-vessel coronary artery disease (CAD) [1]. In most cases it successfully alleviates angina pain and improves early as well as late outcomes [2]. Long-term patency rates of the arterial conduits used for CABG are usually higher as compared to the venous grafts [3].

\author{
Corresponding author: \\ Bartłomiej Perek MD \\ Department \\ of Cardiac Surgery \\ Poznan University \\ of Medical Sciences \\ $1 / 2$ Długa St \\ 61-848 Poznan, Poland \\ Phone/fax: +48 618549085 \\ E-mail: bperek@yahoo.com
}


Adequate arterial conduits, however, are not always available, so the more easily harvested saphenous vein grafts are still widely employed.

At least three principal mechanisms (thrombosis, intimal hyperplasia, and accelerated atherosclerosis) are considered to cause venous graft failure [4]. It results in either an acute stricture just after surgery or progressive narrowing of the graft lumen, eventually followed by complete occlusion observed within months or years after the operation [5]. Although many biochemical and physical stimuli are involved in these processes, there is strong evidence that vascular smooth muscle cells (SMCs) play a crucial role in the pathogenesis of late graft occlusion [6].

On the other hand, vascular aging was shown to have a significant impact on structure and function of the arterial wall [7]. This type of remodelling included a luminal enlargement followed by intimal and medial thickening. The process of arterial wall overgrowth is governed by progressive SMC migration from the tunica media and accumulation in the tunica intima which change of proliferative and apoptotic activity as well as response to growth factors [7]. However, there are only single observations reporting age-related morphological and functional changes of the peripheral veins (e.g. saphenous vein segments) [8].

In general, cardiac surgeons prefer to use the arterial grafts in younger subjects requiring CABG but still accept saphenous vein conduits in the elderly. However, up to now nobody has defined the age limits for venous or arterial grafting. Moreover, no histological parameters which might grade the process of saphenous venous aging have been determined yet.

In view of the above, the aim of the present work was to estimate histological representative features of saphenous vein aging as well as to find the patient age limits which could be used in the future for optimal selection of grafts (arterial or venous) employed in the CABG procedure.

\section{Material and methods}

\section{Study population}

After approval by the Local Ethics Committee (Approval No. 1201/08) and informed consent from the study participants, 110 patients (83 males and 27 females at the age ranging from 46 to 85 years) who underwent CABG between November 2008 and February 2009 were enrolled in the study. They were retrospectively divided into 4 subgroups according to the age at the time of surgery: subgroup A - 50 years and less $(n=19)$, subgroup $B$ - between 51 and $60(n=24)$, subgroup $C$ - between 61 and $70(n=40)$ and subgroup
D - above 70 years old ( $n=27)$. The preoperative demographic and clinical history data are summarized in Table I.

\section{Patient selection}

All the patients selected for CABG on the basis of coronary angiography (Table II) were candidates for inclusion in the study protocol except for subjects (1) aged > 85 years, (2) with body weight $>95 \mathrm{~kg}$, (3) who required emergency surgery due to cardiogenic shock and (4) who were referred directly from the catheterization laboratory with acute complications of percutaneous coronary interventions $(\mathrm{PCl})$.

\section{Operation procedure and sample collection}

The saphenous vein was harvested throughout a full-length thigh incision over its course [9]. Key points included minimal manipulation of the graft ("no-touch" technique), avoiding probing or extensive dilation of the conduit, using low-intensity electrocautery, and control of the branches with stainless-steel vascular clips.

The distal part of the harvested venous segment (approximately $1.5-2 \mathrm{~cm}$ long) was taken for histological study just after vein harvesting. It was carefully rinsed with $0.9 \% \mathrm{NaCl}$ at room temperature, slightly dilated (pressure not exceeding $30 \mathrm{~mm} \mathrm{Hg}$ ) and fixed in freshly prepared Bouin solution. Afterwards the samples stored at $4^{\circ} \mathrm{C}$ were transferred to the Department of Histology and Embryology, where they were embedded in paraffin and cut into 5-6 $\mu \mathrm{m}$ thin sections.

\section{Morphometric analysis}

Each sample of the saphenous vein (taken from separate patients) was cut into 30 paraffin sections. Every fifth section was then stained with haematoxylin and eosin $(\mathrm{H}+\mathrm{E})$ and then analysed using an Olympus BX50 light microscope at the magnification range between $50 x$ and $400 x$.

Briefly, the thickness of the whole venous wall (WallTh), intima (IntTh), media (MedTh) and adventitia (AdvTh) was measured at least six times throughout the vessel circumference at 50x magnification. The average value was then calculated and recorded for further statistical analysis. It was followed by specification of two additional values: MedTh/WallTh and MedAr/WallAr.

To avoid the possibility of results overestimation, cross-sectional areas of the whole venous wall (WallAr) as well as each of its layers were calculated according to the following formulas:

(1) WallAr = OutAr - ILAr,

(2) IntAr = IntELAr - ILAr, 
Table I. Preoperative demographic and clinical data

\begin{tabular}{|c|c|c|c|c|}
\hline Parameter & Group A $(n=19)$ & Group B $(n=24)$ & Group C $(n=40)$ & Group D $(n=27)$ \\
\hline Age (mean \pm SD) [years] & $48.4 \pm 1.3$ & $56.0 \pm 1.1$ & $64.5 \pm 2.6$ & $74.3 \pm 3.8$ \\
\hline Gender $[M / F]$ & $18 / 1$ & $19 / 5$ & 29/11 & $17 / 10$ \\
\hline $\mathrm{BMI}\left[\mathrm{kg} / \mathrm{m}^{2}\right]$ & $30.6 \pm 3.6$ & $29.5 \pm 4.5$ & $29.4 \pm 4.1$ & $27.5 \pm 4.4$ \\
\hline Obesity (BMI > $\left.30 \mathrm{~kg} / \mathrm{m}^{2}\right), n(\%)$ & $10(53)$ & $12(50)$ & $16(40)$ & $7(26)$ \\
\hline \multicolumn{5}{|l|}{ CAD, $n(\%):$} \\
\hline Stable angina & $17(89)$ & $19(79)$ & $34(85)$ & $22(81)$ \\
\hline Unstable angina & $2(11)$ & $5(21)$ & $6(15)$ & $5(19)$ \\
\hline History of infarct & $15(79)$ & $14(58)$ & $28(70)$ & $17(63)$ \\
\hline Previous $\mathrm{PCl}$ & $10(53)$ & $7(29)$ & $13(33)$ & $6(22)^{*}$ \\
\hline Arterial hypertension, $n$ (\%) & $14(74)$ & $19(79)$ & $31(78)$ & $15(56)$ \\
\hline Diabetes mellitus, $n$ (\%): & $6(32)$ & $10(44)$ & $23(58)$ & $14(52)$ \\
\hline Diet & $1(5)$ & $2(8)$ & $1(3)$ & $2(7)$ \\
\hline Oral & $4(21)$ & $4(17)$ & $12(30)$ & $6(22)$ \\
\hline Insulin & $1(5)$ & $6(25)$ & $20(50)^{\#}$ & $6(22) \S$ \\
\hline Hyperlipidaemia, $n$ (\%) & $10(53)$ & $10(42)$ & $17(43)$ & $9(33)$ \\
\hline PVD, $n(\%)$ & $5(26)$ & $7(29)$ & 7 (18) & $10(37)$ \\
\hline Neurological events, $n$ (\%): & 0 & $2(8)$ & $4(10)$ & $3(11)$ \\
\hline TIA & 0 & $1(4)$ & $2(5)$ & $1(4)$ \\
\hline Stroke & 0 & $1(4)$ & $2(5)$ & $2(7)$ \\
\hline Smoking $1, n(\%):$ & $14(74)$ & $17(71)$ & $20(50)$ & $8(30)^{*}$ \\
\hline Active & $8(42)$ & $8(33)$ & $5(13)^{\#}$ & $0 * \$$ \\
\hline History & $6(32)$ & $9(38)$ & $15(38)$ & $8(30)$ \\
\hline Familial history, $n(\%)$ & $11(58)$ & $7(29)$ & $18(45)$ & $5(19)^{*}$ \\
\hline
\end{tabular}

$B M I$ - body mass index, $C A D$ - coronary artery disease, $P C I$ - percutaneous coronary intervention, $P V D$ - peripheral vascular disease, TIA - transient ischaemic attack; ${ }^{1}$ Active considered if smoking cessation up to one year prior to surgery, history if smoking cessation between 10 and 1 year ago; ${ }^{*} p<0.05$ group A vs. group $D,{ }^{\#} p<0.05$ group $A$ vs. group $C,{ } p<0.05$ group $C$ vs. group $D,{ }^{\$} p<0.05$ group $B$ vs. group $D$

Table II. Preoperative coronary angiography

\begin{tabular}{|lcccc|}
\hline Parameter & Group A $(n=19)$ & Group B $(n=24)$ & Group C $(n=40)$ & Group D $(n=27)$ \\
\hline $\begin{array}{l}\text { Coronary angiography, } n(\%): \\
\text { Left main disease }\end{array}$ & $8(42)$ & $9(37)$ & $18(45)$ & $12(44)$ \\
\hline One-vessel disease & 0 & 0 & 0 & $1(4)$ \\
\hline Two-vessel disease & $2(11)$ & $3(13)$ & $4(10)$ & $5(19)$ \\
\hline Three-vessel disease & $9(47)$ & $12(50)$ & $18(45)$ & $9(33)$ \\
\hline
\end{tabular}

(3) MedAr = ExtELAr - IntELAr,

(4) AdvAr = OutAr - ExtELAr,

where IntELAr is the area inside the internal elas-

tic lamina, ILAr - the area inside the vessel lumen, ExtELAr - the area inside the external elastic lamina and OutAr - the area inside the external vessel border.

Afterwards, the number of SMC nuclei within a square of $50 \mu \mathrm{m} \times 50 \mu \mathrm{m}$ was counted at $400 \times$ magnification. The acquired value was then multiplied by 400 and the final result of nuclei number per $\mathrm{mm}^{2}$ was obtained. Finally, the shape (length, thickness and length/thickness ratio) of 6-10 SMC representative nuclei in every individual vessel specimen was assessed at 400× magnification.

\section{Statistical analysis}

All continuous variables were expressed as mean \pm standard deviation. Initially, they were estimated for normality with the Shapiro-Wilk $W$ test. When they complied with a normal distribution, a subsequent analysis of variance (ANOVA) was employed. 
It was then followed by post-hoc comparison of means (Tukey honest significant difference test for unequal number of samples in group - Spjotvoll/Stoline test). Non-parametric continuous variables were compared using the Kruskal-Wallis test. Pearson $\chi^{2}$ test was employed to analyse nominal data. A $p$ value $<0.05$ was regarded as statistically significant. Analyses were carried out using Statistica 9.0 for Windows (StatSoft, Inc., Tulsa, OK, USA).

\section{Results}

Preoperative demographic and clinical data of all the studied patients are summarized in Table I. Some of the study subjects differed significantly in previous $\mathrm{PCl}$ rate or insulin dependent diabetes mellitus incidence. Individual study participants of the successive subgroups did not differ significantly with respect to prevalence of obesity, stable or unstable angina, hypertension, hyperlipidaemia, number of neurological events in the history or peripheral vascular diseases.

According to the histological evaluation of the saphenous vein specimens, both the whole venous wall thickness and its cross-sectional area were found to become systematically smaller in the successive study subgroups (subgroup $A>$ subgroup B > subgroup $C>$ subgroup D). Statistical significance in these differences, however, was found exclusively between subgroups $\mathrm{A}$ and $\mathrm{D}(p<0.05$, Tables III and IV). It was first of all manifested by progressive thinning with aging (atrophy) of the tunica media and significant lowering of the MedTh/WallTh index (Tables III and IV; Figures 1 and 2). What is interesting, the tunica intima and tunica adventitia did not differ significantly between successive study subgroups regarding their thickness or the whole estimated area.

The number of SMC nuclei within the tunica media was revealed to increase in the successive study subgroups (from $3107 \pm 343$ to $3417 \pm 487$ nuclei $/ \mathrm{mm}^{2}$ respectively in groups $A$ and $\left.D\right)$. In addition, the majority of the nuclei in subgroup $D$ were significantly more elongated than in subgroup $A$. Similarly, the length/width index was shown to increase systematically with aging (subgroup $A$ < subgroup $B$ < subgroup $C$ < subgroup D) but statistical significance was reached only between the youngest (subgroup A) and the oldest (subgroup D) study participants (7.94 \pm 0.94 vs. 8.76 $\pm 0.80 ; p<0.05$; Table V).

\section{Discussion}

Our present study, characterizing saphenous vein remodelling, revealed a number of age-related histological and morphometric changes having a potent impact on outcome and long-term followup of CABG patients.

As mentioned in the introduction, there are three distinct mechanisms that are considered to limit the saphenous vein graft patency rate. Early occlusion, recognized up to one month after surgery and involving approximately $15 \%$ of cases, is first of all a result of thrombosis [10]. It is attributable to the operation itself and is due to improper graft harvesting (i.e. damage to the vascular wall, uncontrolled distension, etc.). Interestingly, an inadequate vein-to-artery anastomosis followed by turbulent flow (surgical error, discrepancy in venous to artery diameter), inadequate quality of the recipient artery

Table III. Thickness of whole venous wall and its layers

\begin{tabular}{|lcccc|}
\hline Parameter & Group A $(n=19)$ & Group B $(n=24)$ & Group C $(n=40)$ & Group D $(n=27)$ \\
\hline WallTh $[\mu \mathrm{m}]$ & $437.2 \pm 72.7$ & $424.3 \pm 78.7$ & $413.7 \pm 99.7$ & $386.5 \pm 80.4^{*}$ \\
\hline IntTh $[\mu \mathrm{m}]$ & $51.0 \pm 13.8$ & $50.1 \pm 26.9$ & $64.0 \pm 30.1$ & $55.5 \pm 18.3$ \\
\hline MedTh $[\mu \mathrm{m}]$ & $245.5 \pm 39.8$ & $231.8 \pm 64.7$ & $220.4 \pm 54.7$ & $190.8 \pm 47.1^{*}$ \\
\hline MedTh WallTh & $0.57 \pm 0.08$ & $0.56 \pm 0.08$ & $0.52 \pm 0.08$ & $0.50 \pm 0.09^{\star}$ \\
\hline AdvTh $[\mu \mathrm{m}]$ & $170.6 \pm 57.3$ & $157.9 \pm 47.1$ & $163.3 \pm 37.7$ & $159.6 \pm 47.6$ \\
\hline
\end{tabular}

${ }^{*} p<0.05$ group $A$ vs. group $D$

Table IV. Cross sectional area of the venous wall and its layers

\begin{tabular}{|lcccc|}
\hline Parameter & Group A $(n=19)$ & Group B $(n=24)$ & Group C $(n=40)$ & Group D $(n=27)$ \\
\hline WallAr $\left[\mathrm{mm}^{2}\right]$ & $2.39 \pm 0.60$ & $2.30 \pm 0.76$ & $2.17 \pm 0.73$ & $1.86 \pm 0.70^{\star}$ \\
\hline IntAr $\left[\mathrm{mm}^{2}\right]$ & $0.25 \pm 0.13$ & $0.27 \pm 0.15$ & $0.30 \pm 0.19$ & $0.26 \pm 0.15$ \\
\hline MedAr [mm 2$]$ & $1.05 \pm 0.27$ & $1.13 \pm 0.48$ & $0.98 \pm 0.37$ & $0.78 \pm 0.38^{\star \&}$ \\
\hline MedAr/WallAr & $0.45 \pm 0.09$ & $0.48 \pm 0.08$ & $0.45 \pm 0.07$ & $0.41 \pm 0.08$ \\
\hline AdvAr $\left[\mathrm{mm}^{2}\right]$ & $1.09 \pm 0.44$ & $0.89 \pm 0.32$ & $0.89 \pm 0.33$ & $0.82 \pm 0.28$ \\
\hline
\end{tabular}

${ }^{*} p<0.05$ group A vs. group $D ; \& p<0.05$ group $B$ vs. group $D$ 


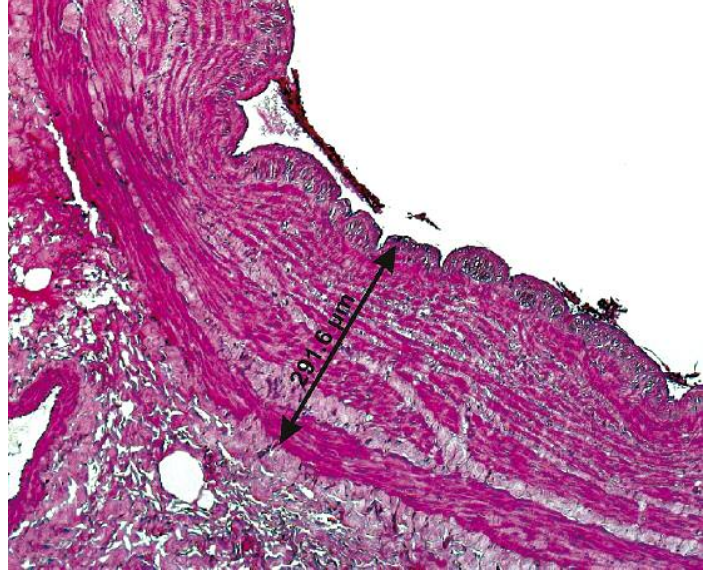

Figure 1. $\mathrm{H}+\mathrm{E}$ stained cross section through the venous wall harvested in group A patient (49-year man). Note presence of thick media (MedTh $291.6 \mu \mathrm{m}$, MedTh/WallTh 0.72, MedAr $0.92 \mathrm{~mm}^{2}$, MedAr/ WallAr 0.56), 200x

(poor runoff phenomenon) as well as too intensive prothrombotic treatment (platelet infusion, protamine administration, etc.) might cause similar early complications $[5,11]$. In addition, there are some supplementary factors predisposing saphenous vein grafts to thrombus formation. These include reduced plasma expression of heparan sulfate and decreased plasma expression of tissue plasminogen activator [12]. Two other mechanisms - hyperplastic neointimal formation followed by media hypertrophy (predominant within months after CABG) and atheroma development (years after surgery) - are less surgery-dependent. Although aggressive vein preparation with high-pressure distension may, to some extent, facilitate these processes [11, 13], it is believed that the biology of the saphenous vein is the primary factor predicting these complexities.

The most intriguing phenomenon of saphenous vein transplants engrafted to the coronary circulation is the process called vein arterialization. It is an unavoidable sequel of physiochemical and hemodynamic changes following vein to arterial system interposition [14]. Vein arterialization is rather an unfavourable process of graft remodelling usually leading to slow but progressive occlusion of the lumen. It is most of all caused by

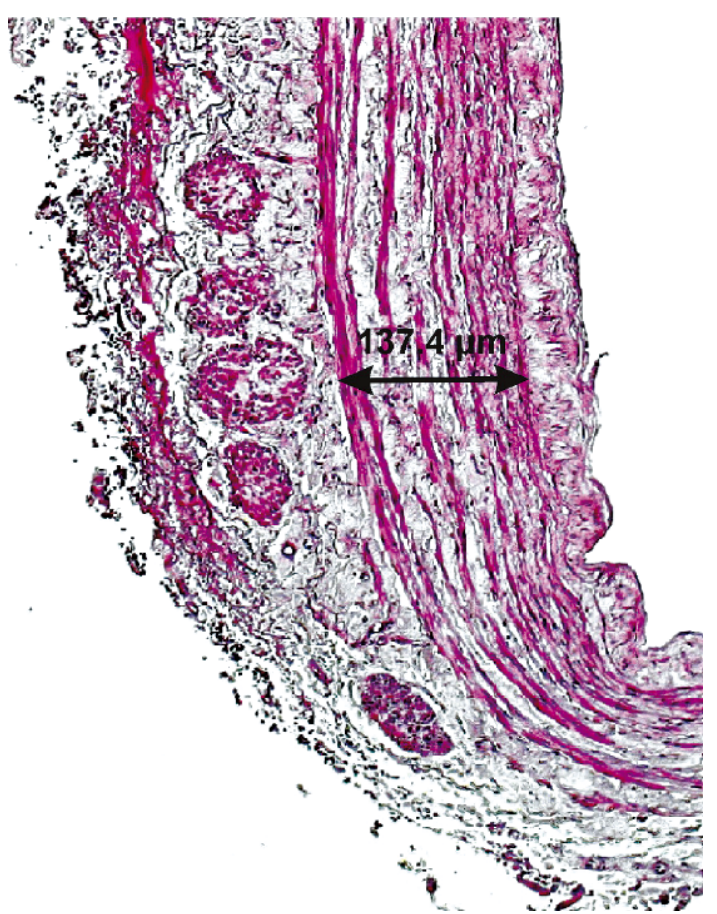

Figure 2. Cross section through venous wall with hypotrophic media harvested in group $D$ patient (71-year woman, MedTh 137.4 $\mu \mathrm{m}$, MedTh/WallTh 0.45, MedAr $0.59 \mathrm{~mm}^{2}$, MedAr/WallAr 0.42), H + E, $200 \times$

the presence of the relatively under-developed venous wall in the high pressure of the arterial circulation [15]. The incontrovertible basis of this sequence is uncontrolled neointimal and medial hypertrophy, leading to the occlusive graft atheroma recognized sometimes many years after surgery [16]. The arterialization process also refers to the unquestionable secretion of growth factors and cytokines, as well as activation of macrophages and platelets giving rise to severe endothelial failure [17]. In addition, some of the effector cells in this process are SMCS, which change their contractile phenotype to a proliferative one, migrate through the internal elastic lamina to the intima, proliferate and secrete extracellular matrix [18].

Smooth muscle cells are the main component of the media, which are arranged in three layers separated by loose connective tissue and elastic fibres [11]. Contrary to the well-known age-associ-

Table V. Number and shape of SMC nuclei in the particular examined groups

\begin{tabular}{|lcccc|}
\hline Parameter & Group A $(n=19)$ & Group B $(n=24)$ & Group C $(n=40)$ & Group D $(n=27)$ \\
\hline SMC nuclei number [nuclei/mm²] & $3107 \pm 342$ & $3205 \pm 435$ & $3223 \pm 611$ & $3416 \pm 486$ \\
\hline Nuclei length $[\mu \mathrm{m}]$ & $13.70 \pm 1.26$ & $14.01 \pm 1.70$ & $14.34 \pm 1.52$ & $14.44 \pm 1.15$ \\
\hline Nuclei width $[\mu \mathrm{m}]$ & $1.75 \pm 0.24$ & $1.78 \pm 0.18$ & $1.77 \pm 0.18$ & $1.73 \pm 0.16$ \\
\hline Nuclei area $\left[\mu \mathrm{m}^{2}\right]$ & $22.01 \pm 3.42$ & $22.76 \pm 3.49$ & $23.18 \pm 2.82$ & $23.10 \pm 2.51$ \\
\hline Length/width index & $7.94 \pm 0.94$ & $8.16 \pm 1.16$ & $8.29 \pm 1.15$ & $8.76 \pm 0.80^{*}$ \\
\hline
\end{tabular}

$S M C$ - smooth muscle cell; ${ }^{*} p<0.05$ group A vs. group D 
ated remodelling in arteries, our study, for the first time, revealed a venous, age-related wall alteration. It has become thinner in the older population, most likely due to the medial atrophy and degeneration of SMCs. Moreover, our findings corresponding to the change of SMC nuclei shape in aging may be of fundamental importance in respect of SMC proliferative activity. It has already been revealed that SMCs exhibiting a more elongated nucleus were associated with a significantly lower proliferation rate [19]. Similarly, other studies reported that a vascular population of SMCs was heterogeneous and manifested at least two morphologically different phenotypes, spindle- and epithelioid-shaped, which resulted in different proliferative and chemotactic activity [20-22]. We are aware of possible bias of the analysis of the cross-sectional histological specimen, but a large number of studied SMC nuclei should minimize its impact on our findings. Thus, it is possible that in the venous segments harvested from older individuals ( $>70$ years old), high representation of the more differentiated SMCs with lower proliferative and chemotactic rates may prevent an unfavourable arterialization process. This finding might be crucial for management with CAD patients undergoing CABG. On the basis of this study we can assume that elderly patients will probably benefit more, in terms of long-term graft patency, from venous aorto-coronary grafts than younger individuals. However, these histological findings must be supported by clinical as well as hemodynamic (coronary angiography) follow-up of CABG patients.

Our study was performed on a relatively small number of patients (group A in particular), which could affect the statistical analysis. Study participants aged $<50$ years represent the smallest subset of patients selected for CABG. Moreover, in these subjects, harvesting arterial bypass grafts is always attempted when possible.

In conclusion, our morphometric studies of saphenous vein grafts used for CABG revealed a progressive, age-related thinning of both the venous wall and the tunica media. Elongation of nuclei of SMCs in the elderly might suggest significant impairment in their migration and proliferation rate. Consequently, individuals aged 70 years and over might benefit clinically more from venous bypass grafts than younger CABG patients.

\section{Acknowledgments}

This study was supported by the State Committee for Scientific Research (grant no. 5958/B/P01/ 2010/38).

\section{References}

1. Serruys PW, Morice MC, Kappetein PA, et al. Percutaneous coronary intervention versus coronary artery bypass grafting for severe coronary artery disease. N Eng J Med 2009; 360: 961-72.

2. Davis KB, Chaitman B, Ryan T, Bittner V, Kennedy JW. Comparison of 15-year survival for men and women after initial medical or surgical treatment for coronary artery disease: a CASS registry study. Coronary Artery Surgery Study. J Am Coll Cardiol 1995; 25: 1000-9.

3. Guru V, Fremes SE, TU JV. How many arterial grafts are enough? A population-based study of midterm outcomes. J Thorac Cardiovasc Surg 2006; 131: 1021-8.

4. Cox JL, Chiasson DA, Gotlieb Al. Stranger in a strange land: the pathogenesis of saphenous vein graft stenosis with emphasis on structural and functional differences between veins and arteries. Prog Cardiovasc Dis 1991; 34: 45-68.

5. Motwani JG, Topol EJ. Aorto-coronary saphenous vein graft disease: pathogenesis, predisposition, prevention. Circulation 1998; 97: 916-31.

6. Johnson JL, van Eys GJ, Angelini GD, George SJ. Injury induces dedifferentiation of smooth muscle cells and increased matrixdegrading metalloproteinase activity in human saphenous vein. Arterioscler Thromb Vasc Biol 2001; 21: 1146-51.

7. Yildiz O. Vascular smooth muscle and endothelial functions in aging. Ann NY Acad Sci 2007; 1100: 353-60.

8. Pascual G, Mendieta C, Mecham RP, Sommer P, Bellón JM, Buján J. Down-regulation of lysyl oxydase-like in aging and venous insufficiency. Histol Histopathol 2008; 23: 179-86.

9. Nowicki M, Buczkowski P, Miśkowiak B, Konwerska A, Ostalska-Nowicka D, Dyszkiewicz W. Immunocytochemical study on endothelial integrity of saphenous vein grafts harvested by minimally invasive surgery with the use of vascular Mayo stripers. A randomized controlled trial. Eur J Vasc Endovasc Surg 2004; 27: 244-50.

10. Bourassa MG, Campeau L, Lespérance J, Grondin CM. Changes in grafts and coronary arteries after saphenous vein aortocoronary bypass surgery: results at repeat angiography. Circulation 1982; 65: 90-7.

11. Hassantash SA, Bikdeli B, Kalantarian S, Sadeghian M, Haleh A. Pathophysiology of aortocoronary saphenous vein bypass graft disease. Asian Cardiovasc Thorac Ann 2008; 16: 331-6.

12. Peykar S, Angiolillo DJ, Bass TA, Costa MA. Saphenous vein graft disease. Minerva Cardioangiol 2004; 52: 379-90.

13. Angelini GD, Passani SL, Breckenridge IM, Newby AC. Nature and pressure dependence of damage induced by distension of human saphenous vein coronary artery bypass grafts. Cardiovasc Res 1987; 21: 902-7.

14. Dobrin PB. Mechanical factors associated with the development of intimal and medial thickening in vein grafts subjected to arterial pressure. A model of arteries exposed to hypertension. Hypertension 1995; 26: 38-43.

15. Zou Y, Dietrich H, Hu Y, Metzler B, Wick G, Xu Q. Mouse model of venous bypass graft arteriosclerosis. Am J Pathol 1998; 153: 1301-10.

16. Domanski MJ, Borkowf CB, Campeau L, et al. Prognostic factors for atherosclerosis progression in saphenous vein grafts: the postcoronary artery bypass graft (Post-CABG) trial. Post-CABG Trial Investigators. J Am Coll Cardiol 2000; 36: 1877-83.

17. Davies MG, Hagen PO. Pathobiology of intimal hyperplasia. Br J Surg 1994; 81: 1254-69.

18. Casscells W. Migration of smooth muscle and endothelial cells: critical events in restenosis. Circulation 1992; 86: 723-9. 
19. Thakar RG, Cheng Q, Patel S, et al. Cell-shape regulation of smooth muscle cell proliferation. Biophys J 2009; 96: 3423-32.

20. Hao H, Gabbiani G, Bochaton-Piallat ML. Arterial smooth muscle cell heterogeneity: implications for atherosclerosis and restenosis development. Arterioscler Thromb Vasc Biol 2003; 23: 1510-20.

21. Li S, Fan YS, Chow LH, et al. Innate diversity of adult human arterial smooth muscle cells: cloning of distinct subtypes from the internal thoracic artery. Circ Res 2001; 89: 517-25.

22. Wang Z, Rao PJ, Castresana MR, Newman WH. TNFinduces proliferation or apoptosis in human saphenous vein smooth muscle cells depending on phenotype. Am J Physiol Heart Circ Physiol 2005; 288: 293-301. 